\title{
New Algorithm based on the Hough Transform for the Analysis of Pulsed Thermographic Sequences
}

\author{
D.A. González ${ }^{\mathrm{a}, \mathrm{b}}$, C. Ibarra-Castanedo ${ }^{\mathrm{b}}$, J.M. López-Higuera ${ }^{\mathrm{a}}$, X. Maldague ${ }^{\mathrm{b}}$ \\ ${ }^{\text {a}}$ Photonic Engineering Group, University of Cantabria, \\ ETSIIT - Avda. Los Castros s/n, 39005 Santander, Spain \\ ${ }^{b}$ Computer Vision and Systems Laboratory, Université Laval, \\ Québec, Canada G1K 7P4 \\ e-mail: dagonfer@teisa.unican.es
}

Keywords:

Pulsed Phase Thermography, Hough Transform, defect detection, defect characterization

\begin{abstract}
The automatic detection of subsurface defects has become one desired goal in the application of Non Destructive Techniques. A new algorithm based on the Hough Transform, is proposed to reduce human intervention to a minimum by Pulsed Thermographic. The final result provided by this algorithm is an image showing the different defects without having attended to parameters so determinant in other algorithms as the delayed time of the first image or any subjective point of view in the analysis.
\end{abstract}




\section{Introduction}

In recent years, Infrared Thermography (IT) has been extensively used in the Non Destructive Evaluation and Testing (NDT\&E) scene. Although various methods have been developed around the world to improve and widen the use of $\mathrm{IT}^{1}$, the human intervention is still necessary to interpret the thermographic sequences. Some previous works have given good results making the analysis of such sequences as independent as possible on the operator's point of view ${ }^{2,3}$. It is obvious that a priori knowledge of the specimen is fundamental most of the time. Nevertheless, in occasions it is sufficient to know whether the thermal decaying profiles at the surface can be modeled as a 1-D semi-infinite homogeneous sample or not. This fact helps to the analysis because the transient heat flow into the body can be quite well approximated by a simplification of the three-dimensional differential equation called Fourier diffusion equation ${ }^{4}$. This simplification characterizes the heat conduction in the body as a function, which relates linearly the logarithm of the temperature increase with the logarithm of the time that the process takes ${ }^{5}$.

Let us consider the inspection of a semi-infinite homogeneous specimen under Pulsed Thermography (PT). The heat transfer at the surface follows a linear decay in a logarithmic space, approximating a -0.5 slope during all the inspection time for a nondefective or sound area, $S_{a}$. On the contrary, thermal decay for a defective area diverges from this behavior. In Figure 1, it is shown the different temporal histories for pixels belonging to defective and non-defective areas. 



b) Temporal evolutions

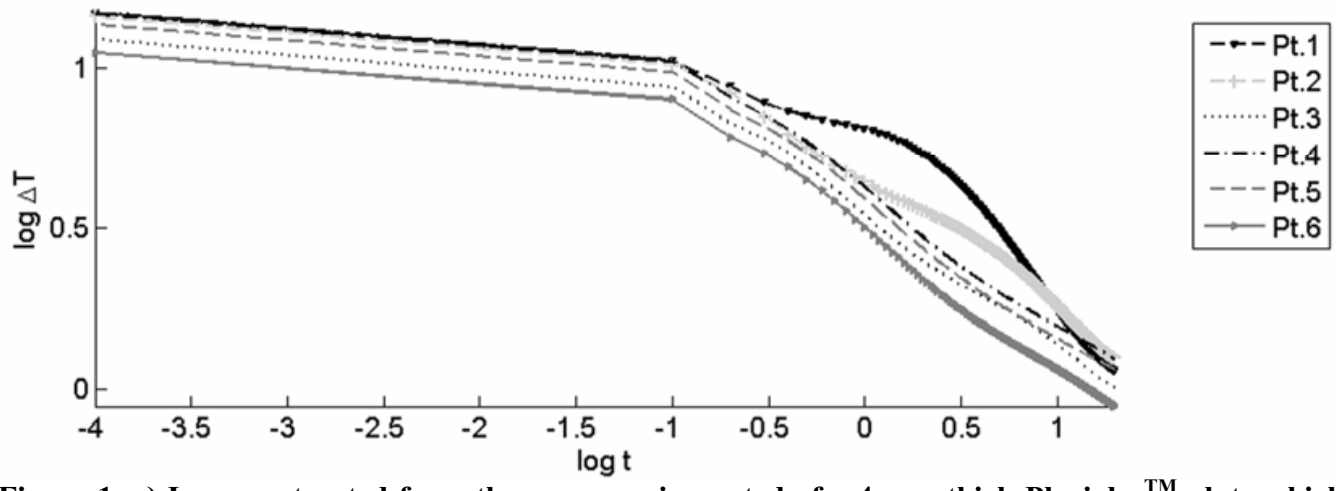

Figure 1. a) Image extracted from the sequence inspected of a $4 \mathbf{m m}$-thick Plexiglas ${ }^{\mathrm{TM}}$ plate which contains $610 \mathrm{~mm}$-diameter flat-bottom circular-shaped holes (localized at depths: 1, 1.5, 2, 2.5, 3, $3.5 \mathrm{~mm}$ ). b) Temporal evolution of contrast temperature for the selected points in a).

The analysis of a thermographic sequence for the detection of subsurface defects can be reduced to the identification of the -0.5 slope in the surface temperature decay for each pixel within the image. Using traditional techniques, which come from the field of computer vision ${ }^{6}$, an algorithm can be developed in order to look for the -0.5 slope in the temporal temperature decay profiles of each pixel. Next sections resume the features of a new algorithm based on Hough Transform, which has been implemented to help in the analysis of thermographic sequences. Results for a Plexiglas ${ }^{\mathrm{TM}}$ specimen are also presented showing the independence on the subjectivity added by different operator's point of view.

\section{Hough Transform in PPT}

The Hough Transform (HT) is a standard tool in image analysis that allows recognition of patterns in an image space. Basically, this technique finds curves that can be parameterized like straight lines, polynomials, circles, etc., in a suitable parameter space $^{7}$. The HT uses the parametric representation of a line:

$$
\rho=x \cdot \cos \theta+y \cdot \sin \theta
$$

eq. 1

A pixel at coordinates $(x, y)$ is transformed into the parameter space $(\rho, \theta)$ where the variable $\rho$ is the distance from the origin to the line along a vector perpendicular to the line and $\theta$ is the angle between the x-axis and this vector. The HT generates a parameter space matrix whose rows and columns correspond to $\rho$ and $\theta$ values respectively. Each point $\left(\mathrm{x}_{\mathrm{i}}, \mathrm{y}_{\mathrm{j}}\right)$, with $\mathrm{i}, \mathrm{j}=1, \ldots, \mathrm{N}$, is transformed into sinusoidal curves in the $(\rho, \theta)$ plane, as 
those shown in Figure 2. The intersection of all those curves shows the existence of a line in the image.
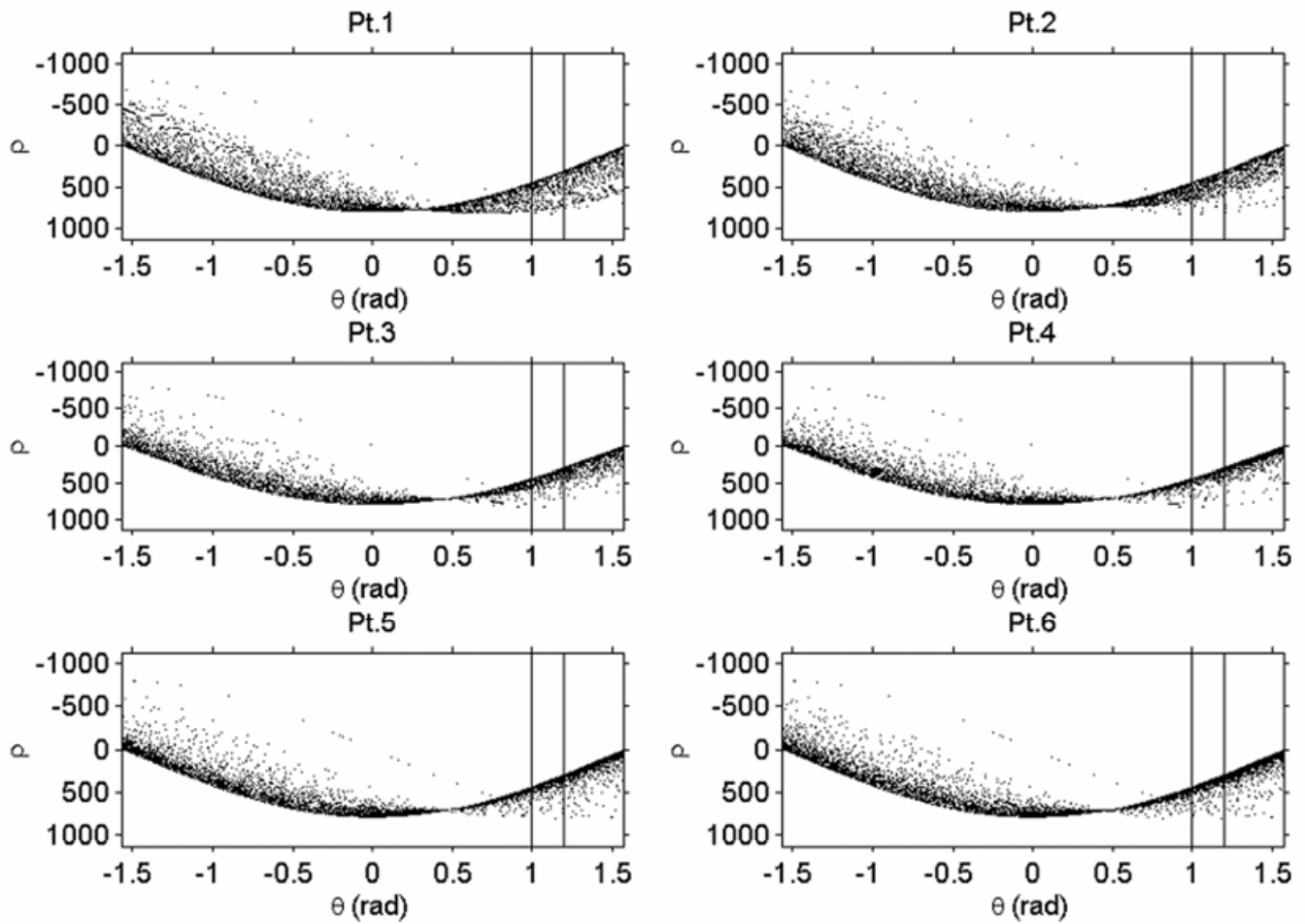

Figure 2. Parameter space $(\rho, \theta)$ for a) a free-defect area pixel b) defective area pixel. Curves are similar in both graphs but the curves spread more in the region $0.6<\theta<0.7$ for the graph $b$ ) because there are more points that do not belong to the same line.

However, assuming a semi-infinite homogeneous material, the goal is not to identify a line in the temporal profile of each pixel, which could be considered as a fitting or regression of data. The goal is to know if that pixel belongs to a defective or a nondefective area. Therefore, locating the number of points than follows a -0.5 slope makes the discrimination. From eq. 1, it can be deduced that the information resides in the column $\theta \approx 1.1071 \mathrm{rad}$.

If all the points in the temporal history agree with a line of -0.5 slope, what should be seen in the Hough space is that all the curves intersect in only a point at the column $\theta \approx 1.1071 \mathrm{rad}$. The coordinate $\rho$ for that point will be the offset of the line. Otherwise, there are as many points in the column as lines with different offsets but the same -0.5 slope in the temporal history. Therefore, analyzing the distribution of the matches in that column selected above, it is possible to know if the pixel belongs to a non-defective area (sharp distribution) or if it belongs to a defective area (broad distribution, broader for deeper defects), as is shown in Figure 3. 
Proc. Vth International Workshop, Advances in Signal Processing for Non Destructive Evaluation of Materials Québec City (Canada),2-4 Aug. 2005. ( X. Maldague ed., É. du CAO (2006), ISBN 2-9809199-0-X


Figure 3. Histograms of values in column $\theta \approx 1.1071 \mathrm{rad}$ for those pixels selected in Figure 1. In each graph the parameters of the fitting fit( $x)=a^{*} \exp \left(-((x-b) / c)^{\wedge} 2\right)$ are given.

Once the HT is applied to each pixel-temperature temporal history, the algorithm evaluates the obtained histograms and divides the pixels depending on the existence or not of the defects. In case that a defect is presented, the depth can be also estimated. Next section contains some of the results obtained using the algorithm together with a discussion about its benefits.

\section{Experimental results}

A 4 mm-thick Plexiglas ${ }^{\mathrm{TM}}$ plate of dimensions $15 \times 15 \mathrm{~cm}$ was stimulated during $15 \mathrm{~ms}$ using two photographic flashes (Balcar FX60). The sample contains $610 \mathrm{~mm}$-diameter flat-bottom circular holes localized at different depths $(1,1.5,2,2.5,3,3.5 \mathrm{~mm})$, see Figure 1.200 thermograms (from $\mathrm{t}=0.1$ to $\mathrm{t}=20 \mathrm{~s}$ ) were recorded after the heating pulse using a Santa Barbara focal plane infrared camera, model SBF125.

Processing of the sequence with the Pulse Thermography Hough Transform Algorithm (PTHTa) it is possible to get results that agree with those obtained through other methodologies shown in Figure 4. 

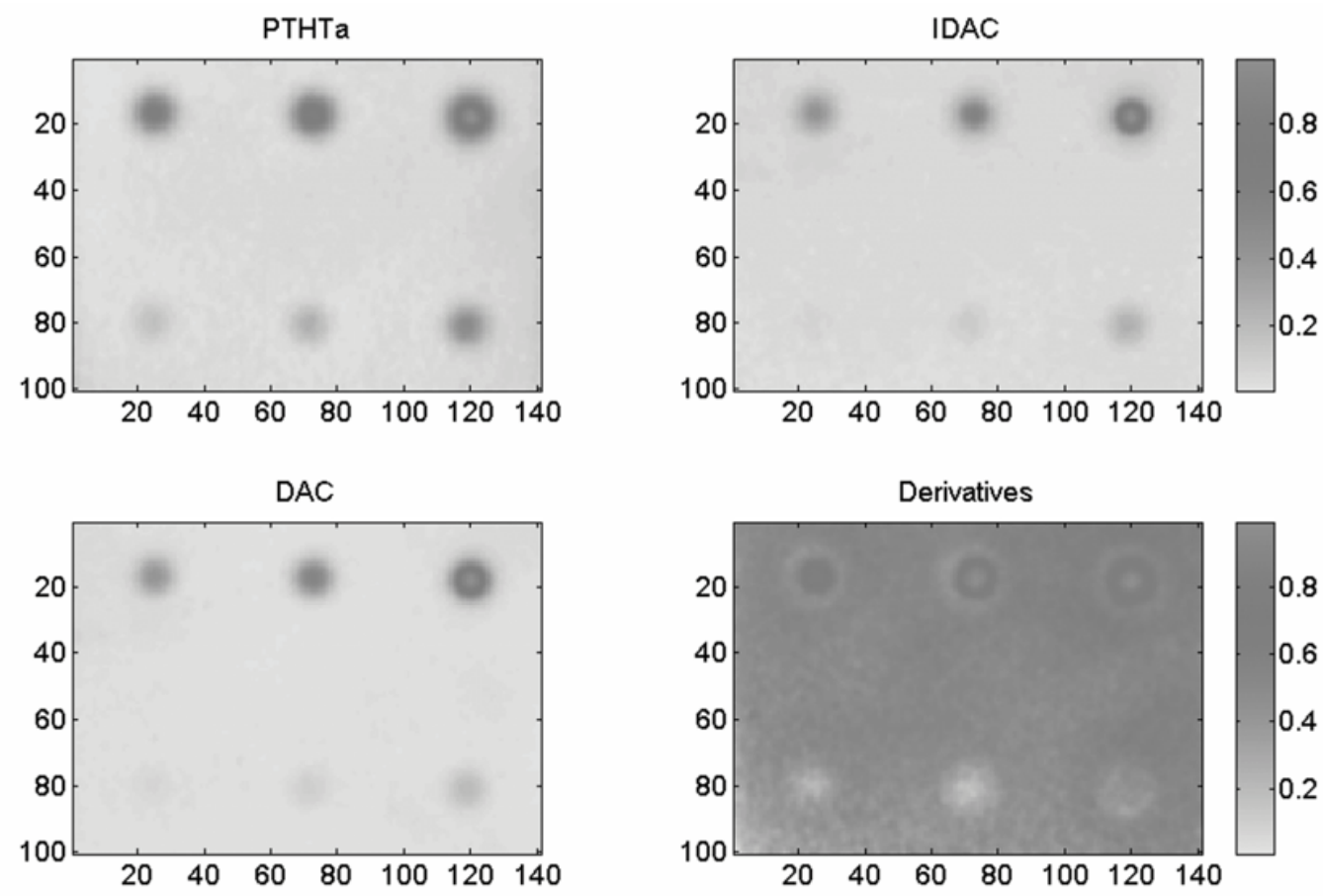

Figure 4. A comparative among different methods of analysis of thermographic sequences is shown. Best contrast images for: a) PTHTa algorithm, here presented b) IDAC algorithm ${ }^{3}$ c) DAC method $^{5}$ d) First and second derivatives ${ }^{8}$.

Although this algorithm is computationally expensive, it provides better-quality contrast images than other algorithms such as IDAC algorithm ${ }^{3}$, DAC method ${ }^{5}$ or the first and second derivatives ${ }^{8}$. For example, the deepest defect is visible by PTHTa while this is not possible by the IDAC method (previous automated algorithm presented by the authors ${ }^{3}$ ). Diffusion is higher at the defects' edges of the as can be seen in the Figure 4, but not as high as the diffusion levels observed with the derivatives method. Furthermore, computing the two-dimensional correlation coefficient between the obtained contrast images, values of 0.93 are achieved. The information according to depth is maintained and also the color scale presents a good correlation with depth as can be seen in Figure 5.

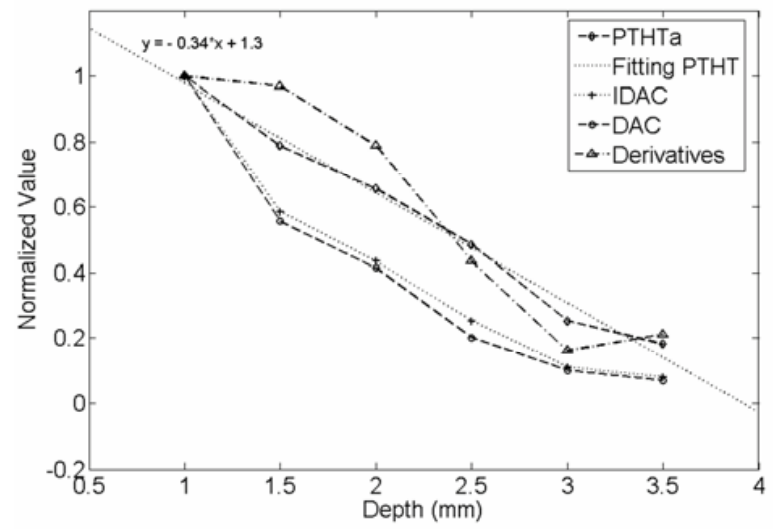

Figure 5. The normalized value of the color scale for each defect on the graphs within Figure 4 is printed versus the depth of the defect. The new PTHT algorithm herein presented shows the best linear behaviour. 
PTHTa makes possible the elimination of errors due to different operator's point of views and so, represents better capabilities in the assessment of specimens. Besides, the selection of a sound area, necessary in most thermographic post processing techniques, is eluded due to be based in a 1-D model. This situation also helps to reduce nonuniformities dues to the heating source, a common problem in active thermography. The influence of the initial time of acquisition, which is a source of incertitude to the measurement and a high non linearity in the log representation of the temporal decay, also is avoided thanks to the fact that dispersed data are not so representative in an algorithm based on occurrences. Therefore, an algorithm absolutely free of human decisions is herein presented.

\section{Conclusions}

A new algorithm based on the Hough Transform is proposed for the analysis of Pulsed Thermographic sequences. The Pulsed Thermography Hough Transform algorithm (PTHTa) provides contrast images free of subjectivity associated with human intervention and avoids the necessity of defining a sound area and establishing the initial time of acquisition. Besides, all the information is contained in just one image and leading to a quantitative estimation of the defect depths. Specimens under inspection should be semi-infinite homogeneous samples because this algorithm is supported on a 1-D Fourier diffusion equation approximation. Experimental works using a Plexiglas ${ }^{\mathrm{TM}}$ specimen were realized showing a good agreement with other semiautomated techniques.

\section{Acknowledgments}

The Spanish Science and Technology Minister under project SiRAS TEC2004-05936C02-02 and the Natural Sciences and Engineering Research Council of Canada have supported this work.

\section{References}

\footnotetext{
${ }^{1}$ X. Maldague, Theory and Practice of Infrared Technology for NonDestructive Testing., John WileyInterscience (2001) p. 684.

${ }^{2}$ D.A. González, C. Ibarra-Castanedo, F.J. Madruga, X. Maldague, Differentiated Absolute Phase Contrast Algorithm for the Analysis of Pulsed Thermographic Sequences, submitted to Infrared Physics \& Technology.

${ }^{3}$ D.A. González, C. Ibarra-Castanedo, M. Pilla, M. Klein, J.M. López-Higuera, X. Maldague, Automatic Interpolated Differentiated Absolute Contrast Algorithm for the Analysis of Pulsed Thermographic Sequences, Proceedings of $7^{\text {th }}$ International Conference on Quantitative Infrared Thermography (QIRT'04), 2004

${ }^{4}$ H.S. Carlslaw, J.C. Jaeger, Conduction of Heat in Solids, Oxford University Press, 2nd edition, 1959.

${ }^{5}$ M. Pilla, M. Klein, X. Maldague, A. Salerno, "New Absolute Contrast for Pulsed Thermography ", Proceedings of QIRT 2002, D. Balageas, G. Busse, G. Carlomagno eds.

${ }^{6}$ W K. Pratt, Digital Image Processing., Wiley, New York (1991) p. 698.

${ }^{7}$ J.C. Russ, The Image Processing Handbook (2nd Edition), CRC Press, Florida (1995), p 674.

${ }^{8}$ R. E. Martin, A. L. Gyekenyesi, S. M. Shepard, "Interpreting the Results of Pulsed Thermography Data," Materials Evaluation, 61[5]: 611-616, 2003.
} 